\title{
Quantum coherence in a model of strongly correlated quantum dots
}

\author{
Xi Dai \\ Department of Physics and Astronomy, Rutgers University, Piscataway, New Jersey 08854-8019, USA \\ Tai-Kai Ng \\ Department of Physics, Hong Kong University of Science and Technology, Clear Water Bay, Kowloon, Hong Kong \\ (Received 19 January 2005; published 2 August 2005)
}

\begin{abstract}
The building up of quantum coherence among coupled mesoscopic objects is studied in the present paper with a model of "ideal" quantum dots. With strong Coulomb repulsion we show that quantum coherence between dots can build up at an energy scale much larger than energy-level spacing if the number of electrons per dot is fractional. The self-consistent ladder approximation (SCLA) is applied to study the quantum coherences in the two-dot and triple-dot systems where we show how quantum coherence manifests itself in the electron spectral function and in the Aharonov-Bohm (AB) effect.
\end{abstract}

DOI: 10.1103/PhysRevB.72.085310

PACS number(s): 73.63.Kv, 72.15.Qm, 71.27.+a

The possibility of establishing quantum coherence between macroscopic objects has been a topic of intense interests for decades since it is central to the foundation of quantum mechanics. ${ }^{1}$ More recently, the problem has attracted attention because of its potential relevance to quantum computation. $^{2}$ The possibility of establishing quantum coherence in realistic mesoscopic quantum dots has been studied both theoretically ${ }^{3-6}$ and experimentally. ${ }^{7-9}$ For small quantum dots where energy level spacing within dots is large compared to temperature, we may approximate a quantum dot as a one level system. Coupled quantum dots can be viewed as "artificial solid" 10 where the single level in different quantum dots are coupled by quantum tunneling and quantum coherence is established at temperature lower than the effective bandwidth of the coupled levels.

Such a simple model is often not applicable to "large" quantum dot systems where temperature is larger than energy level spacing $\left(k_{B} T \geqslant \delta\right)$. For non-interacting electrons electronic transports is dissipative at this regime and quantum coherence between different dots is established only at temperatures $k_{B} T \leqslant \delta .{ }^{11}$ This leads to the usual conclusion that quantum phase coherence is always lost between macroscopic systems where $\delta \rightarrow 0$. The smallest of $\delta$ is a major obstacle to realistic application of large quantum dot systems in quantum computation. This conclusion is not modified by (perturbative) renormalization group treatment of interactions. $^{12}$

There are two major reasons for the loss of phase coherence at $k_{B} T \geqslant \delta$ in quantum dots. The first reason is generic for systems with many quantum transitions (electron hopping across different levels between two different dots) participating more or less equally in a macroscopic process (tunneling of electron from one dot to another). The phase difference between different quantum transitions, when summed over, leads to destructive interference even if the phase variations between different quantum transitions are correlated and not random. ${ }^{13}$ The second reason is applicable to quantum dots with disorder. In this case quantum transitions between different states have intrinsic random phases due to quantum chaos. ${ }^{14}$ We shall show in this paper that for "ideal" quantum dots where quantum chaos is absent quantum coherence be- tween different quantum dots can be established with the help of strong correlation at a new energy scale $k_{B} T_{K} \gg \delta$.

We shall consider a model of $\mathrm{M}$ identical, "ideal" quantum dots coupled by tunnelling processes. In this model the electron number in the system is a key factor determining the low energy physics of the system in the presence of strong coulomb interaction. If the total electron number equals $M \cdot N$ ( $N=$ integer), which is called the commensurate case, the configuration with lowest charging energy has precisely $N$ electrons per dot. Tunnelling processes are suppressed by the charging energy in low temperature. The situation changes when the average electron number per dot is not an integer. In the present paper, we shall consider the simplest situation when the total electron number equals $M \cdot N+1$ (extra electron) or $M \cdot N-1$ (extra hole). In this case we have $M$ degenerate charging states with lowest energy, corresponding to the extra electron (hole) locating on one of the $M$ dots. In the absence of quantum chaos, we shall show that quantum tunnelling will mix these $M$ degenerate charging states nonperturbatively leading to quantum coherence at a temperature $k_{B} T_{K} \geqslant \delta$.

This nonperturbative picture can be obtained most easily by generalizing the Matveev's mapping ${ }^{15}$ of the single quantum dot Coulomb Blockade problem to the Kondo problem where we shall show that by projecting the Hamiltonian into the Hilbert space with $M$ lowest charging states, which is valid for $k_{B} T \ll$ the charging energy $U$, the $M$-dot problem can be mapped to a two-channel SU(M) Coqblin-Schriffer model where the physics of the system can be understood in analogy with Kondo physics. The Kondo temperature is the energy scale below which quantum coherence between quantum dots is established. At temperature well above $T_{k}$, charge dynamics between quantum dots is dissipative. Quantum coherence builds up gradually as $T \rightarrow T_{K}$ and a Kondo resonance peak appears in the electron spectral function at $T$ $\leqslant T_{K}$. By examining the Aharonov-Bohm (AB) effect in a three-dot system, we show that the resonance peak is associated with quantum coherent transport in the system.

We start with the Hamiltonian for the $M$-dot system with $M \cdot N+1$ electrons, 


$$
H_{M-\mathrm{dot}}=H_{c b}+H_{0}+H_{T},
$$

where

$$
H_{c b}=U \sum_{\alpha=1}^{M}\left(\hat{n}_{\alpha}-n_{0}\right)^{2}+V_{\text {gate }} \sum_{\alpha=1}^{M} \hat{n}_{\alpha}
$$

is the Coulomb blockade term with $n_{\alpha}$ being the particle number in the $\alpha$ th dot, $n_{0}$ is electron numbers of a neutral dot, and $V_{\text {gate }}$ is an applied gate voltage to tune the chemical potential,

$$
H_{0}=\sum_{\alpha, k, \sigma} \epsilon_{\alpha k \sigma} C_{\alpha k \sigma}^{+} C_{\alpha k \sigma}
$$

and

$$
H_{T}=\sum_{k, k^{\prime}, \sigma,\langle\alpha \beta\rangle} t_{k, k^{\prime}}^{\alpha \beta} C_{\alpha k \sigma}^{+} C_{\beta k^{\prime} \sigma}+\text { H.c. }
$$

are the kinetic energy within each quantum dot and tunnelling term between them, respectively. We note that similar Hamiltonian has been used to study the transport properties of coupled double-dot system by many authors. 3,5

We now map approximately our Hamiltonian to a quantum rotor Hamiltonian via a generalization of the Matveev's mapping. ${ }^{15}$ The mapping is valid when the total energy level number $\left(N_{\text {total }}\right)$ and total number of electrons $N$ satisfy $N_{\text {total }}$, $N \gg 1$. In the rotor notation, the Hamiltonian can be written as

$$
\begin{aligned}
H_{M-\text { dot }}= & U \sum_{\alpha=1}^{M}\left(\hat{L}_{\alpha}^{z}\right)^{2}+V_{\text {gate }} \sum_{\alpha=1}^{M} \hat{L}_{\alpha}^{z}+\bar{H}_{0} \\
& +\sum_{k, k^{\prime}, \sigma,\langle\alpha \beta\rangle} t_{k, k^{\prime}}^{\alpha \beta} f_{\alpha k \sigma}^{+} f_{\beta k^{\prime} \sigma} \hat{L}_{\alpha}^{+} \hat{L}_{\beta}^{-}+\text {H.c. },
\end{aligned}
$$

where $\bar{H}_{0}=\sum_{\alpha, k, \sigma} \epsilon_{\alpha k \sigma} f_{\alpha k \sigma}^{+} f_{\alpha k \sigma} . \hat{L}_{\alpha}^{z}$ is the angular momentum in $z$ axis of a quantum rotor and represents physically the excess charge on dot $\alpha, \hat{L}^{+}, \hat{L}^{-}$are the raising and lowering operators of a quantum rotor. The physical electron operator at dot $\alpha$ is $C_{\alpha k \sigma}^{+}=f_{\alpha k \sigma}^{+} \hat{L}_{\alpha}^{+}$. The rotor representation is exact if $M$ local constrains, $\Sigma_{l} f_{\alpha, l}^{+} f_{\alpha, l}=n_{0}+\hat{L}_{\alpha}^{z}$, for $i=1, \ldots, M$, are introduced to reproduce the correct Hillbert space. With $N_{\text {total }}$, $N \gg 1$, the above constrains can be treated by a large- $N$ expansion. In the large- $N$ limit, the constraints are satisfied on average by properly choosing the chemical potential, the total fermion number on each dot has fluctuation of order $1 / \sqrt{N}$.

If $V_{\text {gate }}=0$, the ground state without tunnelling term is unique, corresponding to commensurate (neutral) dots. In this case the excited states are gapped and quantum tunnelling term can be treated perturbatively. The quantum coherence between dots is weak.

By applying proper gate voltage we can shift the chemical potential such that states with total charge one are the lowest charging states. In this case we have $\mathrm{M}$ degenerate low energy states, corresponding to the excess electron located at $M$ different dots. Since we are only interested in the low temperature region $k_{B} T \ll U$, we can safely neglect all the other charging states and project the above Hamiltonian to the sub- space containing only the above $\mathrm{M}$ charging states. The projected Hamiltonian can be written as

$$
H_{e f f}=\bar{H}_{0}+\sum_{k k^{\prime}, \alpha \beta, \sigma} t_{k, k^{\prime}}^{\alpha \beta} f_{k \alpha \sigma}^{+} f_{k^{\prime} \beta \sigma}|\alpha\rangle\langle\beta|+\text { H.c. },
$$

where $|\alpha\rangle$ represents a state with the extra electron on dot $\alpha$. For $t_{k, k^{\prime}}^{\alpha \beta}=t$, The above Hamiltonian can be viewed as a two channel SU(M) Coqblin-Schriffer model. In double-dot case, it is also equivalent to two channel anisotropic Kondo mode ${ }^{15,16}$ first proposed by K.A. Matveev to study the charge fluctuation of a single quantum dot connecting to the lead. Here the two lowest energy charging states are equivalent to up and down pseudospin states. Because of degeneracy, a perturbation series in $t$ will diverge logarithmically at low temperature, reflecting the setting up of quantum coherence between the dots below the "Kondo Temperature" $T_{K}$.

The approximation $t_{k, k^{\prime}}^{\alpha \beta} \sim t$ is invalid for "realistic" quantum dots with disorder. In this case $t_{k, k^{\prime}}^{\alpha \beta}$ has totally uncorrelated phases between different tunneling events due to quantum chaos, i.e., $\left\langle t_{k, k^{\prime}}^{\alpha \beta} t_{p, p^{\prime}}^{\alpha^{\prime} \beta^{\prime}}\right\rangle \sim \delta_{k p} \delta_{k^{\prime} p^{\prime}} \delta_{\alpha \alpha^{\prime}} \delta_{\beta \beta^{\prime}}|t|^{2}$ and the problem cannot be mapped into the simple SU(M) CoqblinSchriffer model. The mapping is valid only for "ideal" quantum dots where disorder is absent. For cases with more than one extra electrons (holes), we obtain a similar low energy effective model following the same procedure,

$$
H_{e f f}=\bar{H}_{0}+t \sum_{k k^{\prime}, \alpha \beta, \sigma} f_{k \alpha \sigma}^{+} f_{k^{\prime} \beta \sigma} D_{\alpha \beta}^{\gamma \delta}|\gamma\rangle\langle\delta|+\text { H.c. }
$$

where $D_{\alpha \beta}^{\gamma \delta}=\left\langle\gamma\left|\hat{L}_{\alpha}^{+} \hat{L}_{\beta}^{-}\right| \delta\right\rangle$ and $\langle\gamma|,\langle\delta|$ are states in the set of degenerate charging states with the lowest energy. Notice that the number of degenerate states increases rapidly with increasing number of dots and extra electrons (holes).

In the following we shall apply the self-consistent ladder approximation $^{16-20}$ (SCLA) to solve the above effective Hamiltonian for double- and triple-dots. The SCLA can be viewed as the generalization of the non-crossing approximation for the Kondo Hamiltonian and works very well in all temperature range in the two channel case. Recently the same method has been applied to the problem of charge fluctuation in a quantum box and the results are quite satisfactory compared with Bethe ansatz. ${ }^{16}$ Using the notation and diagrammatic rule in Ref. 20, the diagrams included by SCLA are shown in Fig. 1, where the self energy of the charging state $|\alpha\rangle$ is obtained from the irreducible $T$ matrix of the charging states and fermions. The $T$ matrix is obtained by summing up all the ladder diagrams using the fully dressed Green's function of the charging states, which closes the self consistent integral equations. The SCLA integral equations are solved iteratively and all the details can be found in Refs. 16 and 19. We first examine the electron spectral function in the two-dot system which can be compared with photoemission spectra

$$
G_{\sigma \alpha}\left(i \nu_{n}\right)=\sum_{l, l^{\prime}} \int_{0}^{\beta} e^{i \nu_{n} \tau}\left\langle T L_{\alpha}^{-}(\tau) f_{l \sigma \alpha}(\tau) L_{\alpha}^{+} f_{l^{\prime} \sigma \alpha}^{+}\right\rangle d \tau
$$




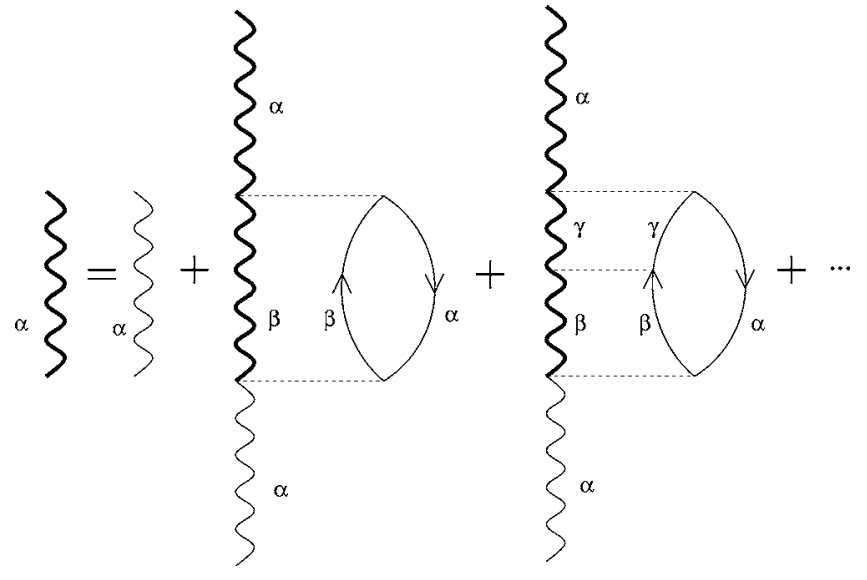

FIG. 1. The diagrams included in SCLA to calculate the renormalized Green's function of $\alpha$ th charging state. The thin and thick wave lines represent the bare and full Green's function of the $\alpha$ th charging states, respectively. The solid lines represent the occupation number of fermions. The dashed lines represent the interaction between fermions and local charging states.

We first consider a double-dot system with $V_{\text {gate }}=0.5, U$ $=1.0, N_{l} t=4.0$, where $N_{l}$ is the total number of levels in a single dot. A semicircle density of states with half band width equals $D=10.0$ is used as the density of states for a single dot. Following Ref. 16 we estimate the Kondo temperature to be $T_{K}=\left(2 D \rho_{0} t\right) \exp \left[-\pi / 4 \rho_{0} t\right]$, which is 0.23 for the above parameters. To calculate the Green's function, we have to include more charging states other than the ones with the lowest energy. The charging states with total charge number $-1,0,1$ are included in our calculation. The generalization of SCLA to include more charging states is quite straightforward. Since the total charge number is a good quantum number in the absence of leads, we can simply apply the SCLA to the subspace with total charge number equals 1 and -1 , respectively, by the above mapping and obtain the renormalized Green's function for each charging states. In the subspace with total charge zero, the lowest energy state is unique and the Green's function can be computed in second order perturbation theory. With the renormalized Green's function for the different charging states, the electron Green's function can be obtained following the diagrammatic rules in Ref. 20. The diagram we used to calculate electron Green's function is shown in Fig. 2. The resulting electron spectral function is shown in Fig. 3. In high temperature where the quantum coherence is lost, a featureless spectra on top of the coulomb gap is found, which reflects the continuous energy levels inside each quantum dot. As temperature decreases, sharp resonance peaks representing the quantum coherent states emerge gradually from the featureless continuum. The presence of the two resonance peaks in the electron spectral function corresponds to transition from charging states -1 to 0 and from 0 to 1 .

To study the quantum interference effect among the quantum dots more carefully, we examine the Aharonov-Bohm $(\mathrm{AB})$ effect in the triple-dot system by connecting two leads to a three-dot system. In the weak lead-system coupling limit, we can treat the quantum tunnelling within the tripledot system by SCLA and treat the lead-system tunnelling by

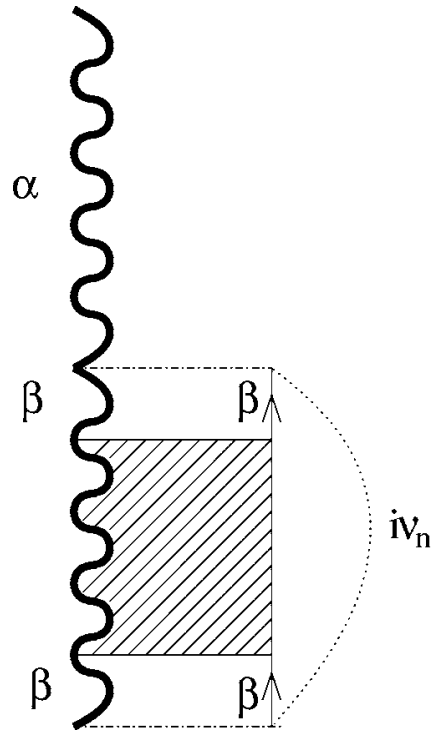

FIG. 2. The diagrams used to calculate physical electron Green's function. The dotted line represents the frequency carried by the green's function and the dot dashed lines represent the creation and annihilation of a physical electron. The shaded area represent all the ladder diagrams in Fig. 1.

first order perturbation. The Master's equation technique ${ }^{21}$ is used to calculate the current flowing through the triple-dot system with a finite voltage added between two leads. As we mentioned above only the lowest three charging states $-1,0,1$ are included in the calculation of conductance. A magnetic flux enclosed by the dots can be included by introducing non trivial phase factors to the tunnelling matrix elements $t^{\alpha \beta} \rightarrow t^{\alpha \beta} e^{i A \alpha \beta}$.

In Fig. 4 we plot our results for the zero bias conductance as the function of gate voltage under different magnetic flux. The parameters we used here are $N_{l} t=2.0, U=1.0, D=10.0$, and $T=0.01$. We observe that the appearance of two resonance peaks in the electron spectral function is reflected in

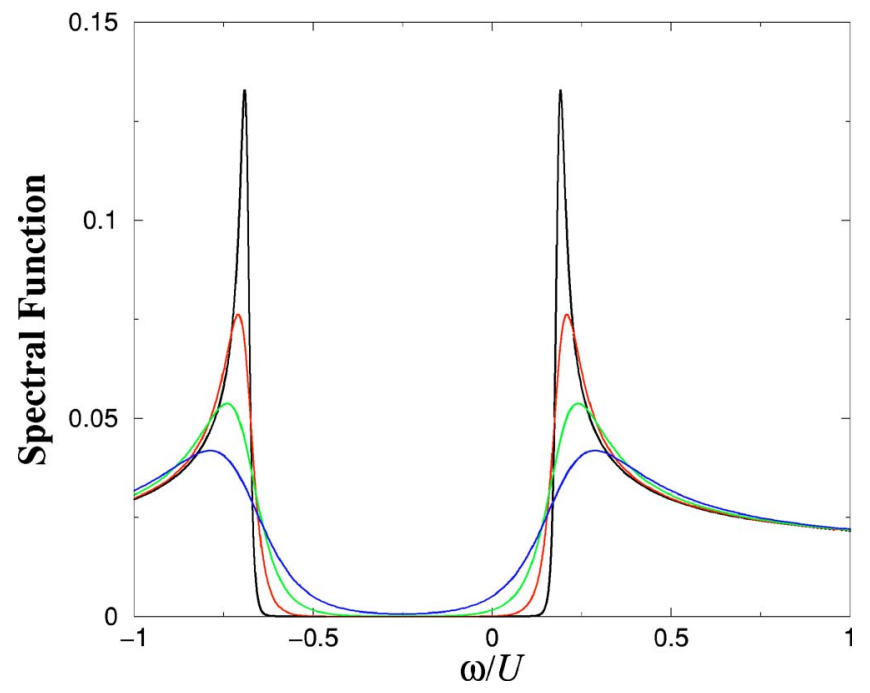

FIG. 3. (Color online) The electronic spectral function for the double-dot system with temperature $T=0.01,0.05,0.1,0.2$, from the top to bottom. 


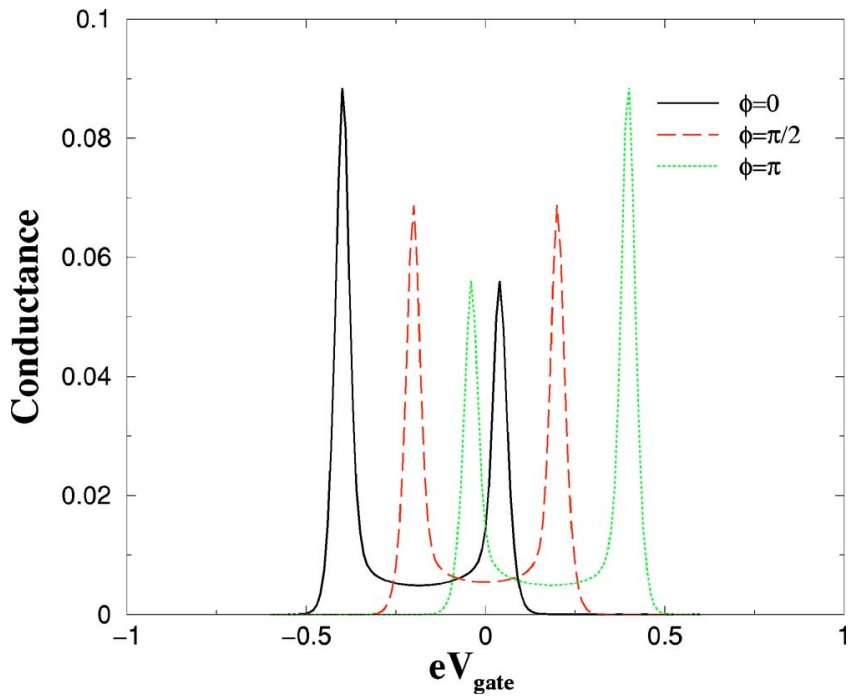

FIG. 4. (Color online) The zero bias conductance as the function of gate voltage under different magnetic flux.

conductance measurement. The resonance peaks move to different gate voltages when magnetic flux changes, i.e., the quantum coherence manifest itself as a giant gate-voltagedependent magnetoresistance effect at low temperature. We also plot the renormalized dc conductance as a function of magnetic flux in Fig. 5 for gate voltage $V_{g}=0.1, N_{l} t=2.0$, $U=1.0$ at different temperatures. At temperature $T=0.01$ we can clearly see two resonance peaks. The resonance feature disappears when the temperature is raised above the Kondo temperature $T_{K} \sim 0.2$ with our parameters. Notice that $g(\Phi) / g(0) \sim O(1)$ for temperature $\geqslant T_{K}$, indicating the lost of quantum coherence.

We emphasize that although the quantum coherence effect we studied here is mathematically similar to the usual Kondo effect, the physical content is rather different. The coherent (pseudo) spin dynamics in usual Kondo effect is map to the charge dynamics in the present problem. It implies that the coherent charge transports we observe here is not the usual quasiparticle transports, but represents a new collective charge transport process. It is a many-body effect established with the help of strong Coulomb interaction and is contrary to the usual perturbative viewpoint where Coulomb interaction is considered as one of the origins of dephasing. As

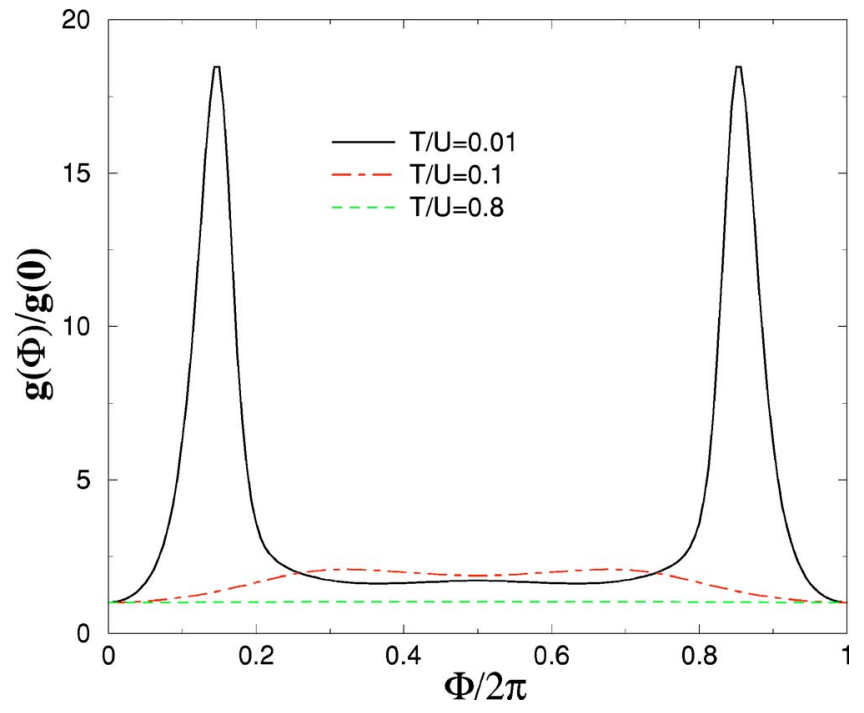

FIG. 5. (Color online) The normalized dc conductance as the function of magnetic flux $\Phi$.

Coulomb repulsion $U$ decreases, it is expected that the Kondo temperature $T_{K}$ will decrease, until $T_{K} \leqslant \delta$, where usual picture of quasiparticle transports recovers.

Summarizing, we show that contrary to usual belief, ${ }^{11,12}$ quantum coherence can be setup between mesoscopic conducting particles at an energy scale $k_{B} T_{K} \gg \delta$ with the help of strong Coulomb interaction-at least for "ideal" particles where disorder is absent. For $M$ coupled identical quantum dots with one extra charge, the low energy effective Hamiltonian is a two-channel SU(M) Coqblin-Schrieffe model and quantum coherence sets up at temperatures below an effective "Kondo temperature" $T_{K}$. To demonstrate, we compute the electron spectral function for a double-dot system using SCLA which shows sharp quasi particle peaks below the Kondo temperature. We also study transports in the triple-dot system where a strong $\mathrm{AB}$ effect is found below the "Kondo temperature," indicating the presence of quantum coherent transports among the dots.

The authors acknowledge helpful discussions with K.A. Matveev. T.K.N. thanks Hong Kong Research Grant Council for support through Grant Nos. HKUST6142/00P and HKUST6159/01P.
${ }^{1}$ See, for example, A. J. Leggett, in Directions in Condensed Matter Physics, edited by G. Grinstein and G. Mazenko (World Scientific, Singapore, 1986).

${ }^{2}$ D. Loss and D. P. DiVincenzo, Phys. Rev. A 57, 120 (1998).

${ }^{3}$ J. M. Golden and B. I. Halperin, Phys. Rev. B 53, 3893 (1996).

${ }^{4}$ I. M. Ruzin, V. Chandrasekhar, E. I. Levin, and L. I. Glazman, Phys. Rev. B 45, 13469 (1992).

${ }^{5}$ K. A. Matveev, L. I. Glazman, and H. U. Baranger, Phys. Rev. B 54, 5637 (1996).

${ }^{6}$ W. Izumida and O. Sakai, Phys. Rev. B 62, 10260 (2000).

${ }^{7}$ F. R. Waugh, M. J. Berry, C. H. Crouch, C. Livermore, D. J. Mar,
R. M. Westervelt, K. L. Campman, and A. C. Gossard, Phys. Rev. B 53, 1413 (1996); F. R. Waugh, M. J. Berry, D. J. Mar, R. M. Westervelt, K. L. Campman, and A. C. Gossard, Phys. Rev. Lett. 75, 705 (1995).

${ }^{8}$ T. Fujisawa, T. H. Oosterkamp, W. G. van der Wie, B. W. Broer, R. Aguado, S. Tarucha, and L. P. Kouwenhoven, Science 282, 932 (1998).

${ }^{9}$ T. H. Oosterkamp, T. Fujisawa, W. G. van der Wiel, K. Ishibashi, R. V. Hijman, S. Tarucha, and L. P. Kouwenhoven, Nature (London) 395, 873 (1998).

${ }^{10}$ W. G. van der Wiel, S. De Franceschi, J. M. Elzerman, T. 
Fujisawa, S. Tarucha, and L. P. Kouwenhoven, Rev. Mod. Phys. 75, 1 (2003).

${ }^{11}$ D. V. Averin and Yu. V. Nazarov, Phys. Rev. Lett. 65, 2446 (1990); I. S. Beloborodov, K. B. Efetov, A. Altland, and F. W. J. Hekking, Phys. Rev. B 63, 115109 (2001).

${ }^{12}$ I. S. Beloborodov, K. B. Efetov, A. V. Lopatin, and V. M. Vinokur, Phys. Rev. Lett. 91, 246801 (2003); D. S. Golubev and A. Z. Zaikin, cond-mat/0402306 (unpublished).

${ }^{13}$ T. K. Ng and H. Y. Cheung, Phys. Rev. B 70, 172104 (2004).

${ }^{14}$ See, for example, K. Efetov, Supersymmetry in Disorder and Chaos (Cambridge University Press, Cambridge, UK, 1997).

${ }^{15}$ K. A. Matveev, Zh. Eksp. Teor. Fiz. 99, 1598 (1991) [Sov. Phys. JETP 72, 892 (1991)].
${ }^{16}$ E. Lebanon, A. Schiller, and V. Zevin, Phys. Rev. B 64, 245338 (2001).

${ }^{17}$ G. Zarand, G. T. Zimanyi, and F. Wilhelm, Phys. Rev. B 62, 8137 (2000).

${ }^{18}$ E. Lebanon and A. Schiller, Phys. Rev. B 68, 155301 (2003); B. Anders, E. Lebanon, and A. Schiller, cond-mat/0311502 (unpublished); S. Florens and A. Rosch, Phys. Rev. Lett. 92, 216601 (2004).

${ }^{19}$ S. Maekawa, S. Takahashi, S. Kashiba, and M. Tachiki, J. Phys. Soc. Jpn. 54, 1955 (1985).

${ }^{20}$ N. E. Bickers, Rev. Mod. Phys. 59, 845 (1987).

${ }^{21}$ L. I. Glazman and R. I. Shekhter, J. Phys.: Condens. Matter 1, 5811 (1989). 